\title{
Remission in SLE: closing in on the target
}

\author{
Ronald F van Vollenhoven, ${ }^{1}$ Alexandre Voskuyl, ${ }^{2}$ Eric Morand, ${ }^{3}$ \\ Cynthia Aranow ${ }^{4}$
}

I'm in complete remission. I'm alive and well.

Gene Wilder

Over the past decades, the concept of 'remission' has emerged as a moniker for the disease state one would ideally like to achieve when a 'cure'-the ultimate goal of medical intervention-cannot realistically be hoped for. Originally used in oncology to describe the absence of detectable tumour, remission has become an important concept among medical specialties treating autoimmune inflammatory diseases. In some of these disease areas, three distinct processes have taken place:

- The term remission was introduced into the parlance of the specialty area, so that physicians, researchers and patients would use the term to describe the state they wished to achieve.

- Remission was specifically defined for each disease; for example, in rheumatoid arthritis (RA) a preliminary American College of Rheumatology (ACR) definition was published in $1981,{ }^{1}$ followed by a definition based on the disease activity score or other disease activity indices, and finally by a joint ACR and European League Against Rheumatism (EULAR) definition ${ }^{2}$; similar developments took place in other chronic inflammatory disease areas, such as inflammatory bowel disease. ${ }^{3}$

- Remission was codified as the explicit target of therapeutic interventions, again most notably in RA where $\mathrm{ACR}^{4}$ and EULAR $^{5}$ guidance as well as the 'treat-to-target' work force $^{6}$ have expressed remission as the goal of therapy for most patients.

\footnotetext{
${ }^{1}$ Department of Medicine, The Karolinska Institute, Stockholm, Sweden; ${ }^{2}$ Department of Rheumatology, Amsterdam Rheumatology and Immunology Center ARC, Amsterdam, The Netherlands; ${ }^{3}$ School of Clinical Sciences, Monash University, Clayton, Victoria, Australia; ${ }^{4}$ Center for Autoimmune and Musculoskeletal, The Feinstein Institute for Medical Research, Manhasset, New York, USA

Correspondence to Dr Ronald F van Vollenhoven Department of Medicine, The Karolinska Institute, ClinTRID, The Karolinska University Hospital D1:0, Stockholm 17176, Sweden; ronald.van.vollenhoven@ki.se
}

Needless to say, these three developments have strongly influenced each other. Thus, the existence of specific and quantitative definitions of remission made it possible to articulate it as a therapeutic target, and has made the term remission a topic of discussion in many scientific publications and in patient-physician encounters.

In systemic lupus erythematosus (SLE), the concept of remission has also been discussed extensively. Remission in SLE is widely understood as a desirable disease state that should be associated with optimal health-related quality of life and a good prognosis. It is remarkable that while remission has been used to describe a favourable clinical state for patients with SLE since at least the $1970 \mathrm{~s}^{7}$ there has not yet been an agreed-upon definition of remission in SLE. As reviewed in a recent publication, ${ }^{8}$ there are a number of different ad hoc definitions of remission that have been used in clinical trials and observational studies (table 1 ).

Accurately defining remission in SLE would serve multiple purposes. It would facilitate many types of clinical research, including epidemiological studies, health economic investigations and clinical trials, leading to standardised cohort descriptions, valid interstudy comparisons and perhaps better trial outcomes. A validated definition of remission could also facilitate communication between healthcare provider and patient, and would be useful in education.

Additionally, SLE is an extraordinarily heterogeneous disease with multiple clinical manifestations, and this heterogeneity has greatly hindered agreement on methods for quantification of disease activity; patients in remission or with low disease activity are clinically (and perhaps mechanistically) more homogeneous than those with active disease, ${ }^{23}$ potentially permitting simpler definitions of these clinical states (illustrated in figure 1). These patients may have achieved an immunological state of tolerance, and therefore, studies of patients with SLE in remission are likely to result in better understanding of the disease.

Recently, the treat-to-target for SLE (T2T/SLE) initiative established international consensus on an approach to the therapy of lupus based on (1) identifying an appropriate target for each patient, (2) directing therapy towards achieving this target and (3) reassessing the target, and if needed changing the treatment. ${ }^{24}$ The T2T/SLE recommendations identified 'remission of systemic symptoms and organ manifestations' as one of the main therapy targets in SLE. However, the panel recognised that no generally accepted definition of remission in SLE exists today, and therefore, the T2T/SLE task force identified the definition of remission as a research priority for this disease. In response, an initiative to achieve consensus on a definition of remission in SLE was undertaken by a large multiparty international task force (DORIS-Definition Of Remission In SLE); the work of this task force has been presented at the 2015 EULAR congress and is being prepared for publication. ${ }^{25}$ The task force agreed that remission in SLE can conceptually be described as a desirable disease state for patients with, at the very least, the absence of major symptoms and signs of SLE. It is conceived of as distinct from a cure and also meaningfully different from a low disease activity state, including the lupus low disease activity state (LLDAS) that has recently been proposed by the Asia-Pacific Lupus Collaboration. ${ }^{26}$ Importantly, for future work in this area, it is recognised that LLDAS and remission are states associated with reduced morbidity and mortality.

Further discussions involving SLE experts and patients identified four critical domains with regard to which definitions of remission are divergent and no clear consensus exists: clinical disease activity, serological activity, duration and treatment (figure 2).

Thus, a definition of remission could require complete absence of clinical disease activity without any signs or symptoms indicative of SLE activity-or alternatively, a certain minimal amount of symptomatology might be accepted. For comparison, the proposed definition of remission in paediatric $\mathrm{SLE}^{27}$ allows certain symptoms, such as mild fatigue, mild myalgia, mild alopecia; the aforementioned remission in RA allows one swollen and one tender joint. As a practical approach, a validated index can be used to define the clinical activity aspect of remission, and this approach has dominated the literature over the past several years. Some different proposals are: systemic lupus disease activity index (SLEDAI) $<2$, 'clinical SLEDAI' (ie, disregarding serology $)=0$, clinical ECLAM $=0$ or having BILAG categories D and E only. 
Table 1 An overview of definitions of remission in SLE used in the literature

\begin{tabular}{|c|c|c|c|c|c|}
\hline Author(s) & Year & Remission definition & $\begin{array}{l}\text { Serological } \\
\text { activity } \\
\text { permitted }\end{array}$ & $\begin{array}{l}\text { Duration of } \\
\text { remission } \\
\text { required }\end{array}$ & $\begin{array}{l}\text { Treatments } \\
\text { permitted }\end{array}$ \\
\hline Dubois $^{9}$ & 1956 & Based on the rheumatologist's impression & Not specified & No & Not specified \\
\hline Dubois and Tuffanelli ${ }^{10}$ & 1964 & Based on the rheumatologist's impression & Not specified & No & Not specified \\
\hline Gladman et al ${ }^{11}$ & 1979 & Asymptomatic patient & Yes & No & None \\
\hline Tozman et al ${ }^{12}$ & 1982 & Absence of clinical manifestations of disease & No & No & None \\
\hline Heller and Schur ${ }^{13}$ & 1985 & Asymptomatic without active organ involvement & No & No & $\begin{array}{l}\text { Antimalarials and } \\
\text { low-dose } \\
\text { glucocorticoids }\end{array}$ \\
\hline LeBlanc et $a l^{14}$ & 1994 & Clinical SLEDAI $=0$ & Yes & $\begin{array}{l}\geq 3 \text { consecutive } \\
\text { clinic visits }\end{array}$ & Any \\
\hline Drenkard et $a l^{15}$ & 1996 & Lack of disease activity permitting SLE treatment withdrawal & Yes & $\geq 1$ year & None \\
\hline Barr et $a l^{16}$ & 1999 & Clinical SLEDAI $=0$ or $\mathrm{PGA}<1.0$ & Yes & $\geq 1$ year & Not specified \\
\hline Formiga et $a l^{17}$ & 1999 & Lack of disease activity permitted SLE treatment withdrawal & Yes & $\geq 1$ year & None \\
\hline Swaak et al ${ }^{18}$ & 1999 & Absence of disease-related signs with no need for treatment & Not specified & No & None \\
\hline Urowitz et al ${ }^{19}$ & 2005 & Clinical SLEDAI $=0$ & Yes & $\geq 5$ years & None \\
\hline Nossent et $a l^{20}$ & 2010 & Physician assessed & Not specified & No & Not specified \\
\hline Steiman et $a l^{21}$ & 2010 & Clinical SLEDAI-2K=0 & Yes & $\geq 2$ years & Antimalarials only \\
\hline Conti et $a l^{22}$ & 2012 & Clinical SLEDAI-2K=0 & Yes & $\geq 2$ years & Antimalarials only \\
\hline
\end{tabular}

Adapted from Steiman et al. ${ }^{8}$

PGA, patient global assessment; SLE, systemic lupus erythematosus; SLEDAl, systemic lupus disease activity index.

Serological activity, understood by most as the demonstration of antidoublestranded DNA antibodies and/or hypocomplementaemia attributable to complement activation, has been included in some remission definitions, but not in others. A state of serological activity with clinical quiescence (SACQ) has been defined where serological, but not clinical, activity is permitted in a patient off therapy other than antimalarials;

persistent SACQ has been associated with improved outcomes. ${ }^{28}$

Duration of remission has emerged as a particularly thorny concept with which to grapple in defining remission in SLE. Because SLE, in contrast to other chronic autoimmune diseases, may have a relapsing-remitting pattern, ${ }^{16}$ it is obvious that remission achieved at only one given point in time may have limited clinical relevance. However, recent data suggest that the

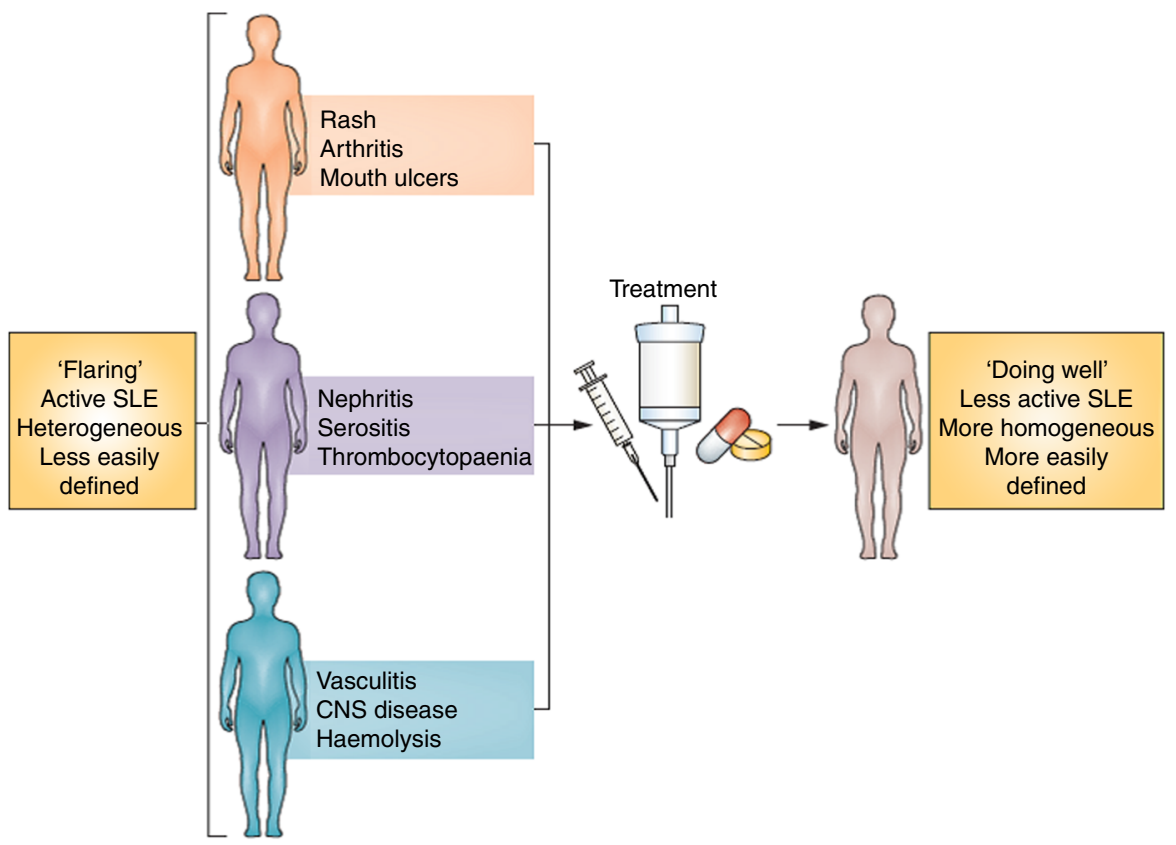

Figure 1 While systemic lupus erythematosus (SLE) is an extraordinarily heterogeneous disease, the patients who are in low disease activity or in remission present with a much less heterogeneous, more readily described clinical state. Reproduced, with permission, from Franklyn et al. ${ }^{23}$ relapsing-remitting pattern is stable in time only in a small subset of patients. ${ }^{29}$ Moreover, definitions of remission in other chronic diseases do not require persistence of the remission state, and the utility of definitions of remission in clinical trials will be limited if they explicitly require a prolonged duration. We consider that omitting a prespecified duration from a definition of remission will allow the effects of various durations to be studied, potentially leading to identification of a threshold duration of remission to seek in a treat-to-target approach in order to positively impact on outcomes.

The fourth component of a remission definition is treatment. Because many feel that antimalarial medications should be considered as long-term maintenance therapy for patients with SLE-even if they have achieved remission-it is clear that treatment with antimalarials cannot preclude the patient from being considered to be in remission. On the other hand, patients who are treated with moderate-dose or high-dose corticosteroids cannot be considered to be in remission even if they would fulfil other criteria for remission. The main argument for this is of course the well-established adverse health consequence of long-term moderate-dose to high-dose corticosteroid treatment. However, it should be noted that definitions of remission in other autoimmune diseases, including $\mathrm{RA}^{2}$ and Crohn's disease, ${ }^{3}$ do not exclude the chronic use of specific antirheumatic medications, immunosuppressives or biologics. 


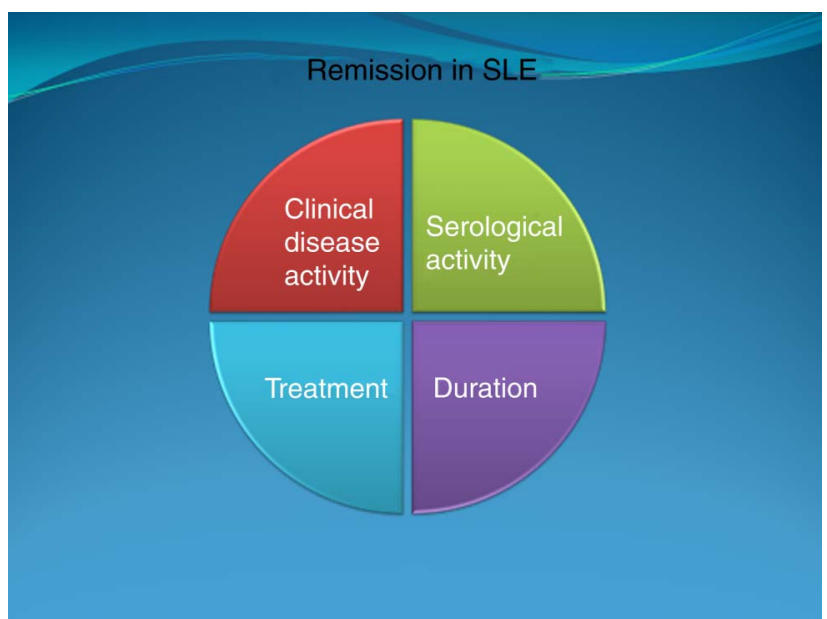

Figure 2 Four domains were considered as critical for defining remission in systemic lupus erythematosus (SLE): clinical activity, serological activity, duration and treatment. ${ }^{25}$

Given these considerations, there are still considerable differences between patients who are clinically in remission, but whose disease requires low-dose corticosteroids, immunosuppressives and/or biologics, and those whose only treatment might be limited to maintenance antimalarials. There have been many approaches to this distinction, including the use of different modifiers and terms: 'complete remission', 'partial remission', 'clinical remission', 'serological remission', 'lupus under control' and 'inactive disease'. The recently convened 'DORIS' task force supported the standardised use of two terms only: complete remission and clinical remission. ${ }^{25}$

Zen $e t a l^{30}$ present analyses that provide interesting new insights into the questions surrounding remission in SLE. The focus of their studies is on patients in prolonged remission, with analyses of 224 Caucasian patients with SLE diagnosed between 1990 and 2009, and who during a 5-year period between 2009 and 2013 were followed at least three times a year at the registry of patients with SLE at the University of Padua in Italy. The authors chose to apply a definition of remission based on the SLEDAI-2K with three levels: complete remission, having no clinical activity, no serological activity and no treatment (other than antimalarials); clinical remission off corticosteroids, where serological activity and stable immunosuppressive therapy are allowed and clinical remission on corticosteroids, where up to $5 \mathrm{mg}$ daily of prednisone is allowed as well as immunosuppressive therapy. Each definition of prolonged remission required that the patient achieved this state for the full 5 years of follow-up. Moreover, because all versions of the SLEDAI contain several 'holes'-organ systems that can be affected by SLE disease activity, but that are not assessed in determination of the SLEDAI, these investigators specifically required that the patient could not have evidence of myelitis, gastrointestinal lupus or haematological lupus activity (the latter being only partially covered by the SLEDAI) to be considered in remission.

Despite these rather stringent requirements, prolonged remission in this cohort was not unusual, $37 \%$ of patients achieving at least the 'clinical remission on corticosteroids', while a complete remission by the above definition was more rare, seen in $7.1 \%$ of patients. Nonetheless, even the latter is considerably higher than the level of complete remission found by Steiman et $a l .{ }^{8}$ Differences in the definitions used and in the populations included in the cohorts could well explain the differences.

Zen et al then address the consequences for the patient of having prolonged remission or not. They show that the development of damage (which could be related to the disease itself or to its treatment, and which was measured using the systemic lupus erythematosus international collaborating clinics (SLICC) damage index ${ }^{31}$ ) was significantly higher among patients who did not achieve a remission compared with those who did at any of their three levels. Thus, only 3 out of 16 $(19 \%)$ patients who were in complete remission and 6 out of $33(18 \%)$ of those in clinical remission off corticosteroids accrued damage (these two groups differed, in effect, by having positive serology or not; and it is of interest that in this study they behaved similarly); in contrast, 13 out of 35 (37\%) patients who were in remission, but on corticosteroids accrued damage and, as expected, the highest risk for damage was seen in 'unremitted patients', those who did not reach any state of prolonged remission where
72 out of 140 (51\%) accrued damage. Overall, these results support the 'construct validity' of their proposed definitions of remission, as a definition of remission should identify patients with a better prognosis.

This study also examined features of disease that associated with achievement of remission, and report that patients with vasculitis, glomerular disease or haematological disease were less likely to achieve prolonged remission. This stands in contrast to studies by Urowitz et $a l^{19}$ and Steiman et $a l^{8}$ who did not find a difference in disease features between patients who did or did not achieve remission. The current study suggests that patients achieving remission may have a fundamentally different phenotype than those who do not remit, while the results of previous studies imply that remission is a state that may, at least potentially, be achieved by any patient with lupus. It will be important to understand the reasons behind these discordant findings.

Thus, Zen et al present very interesting data and they can be complimented for having tackled an important and timely subject. Their use of author-specified definitions of various levels of remission appears reasonable, but underscores the need to arrive at more widely agreedupon, internationally established definitions of remission; current thinking is gravitating towards one definition for complete remission-similar to the one used by Zen et al-and one for clinical remission, which corresponds to the clinical remission on corticosteroids in the current study. Whether the inclusions of a serological requirement is useful remains to be determined in future studies, but the analyses presented here suggest that perhaps this is of lesser importance. In addition, the dose of corticosteroids chosen in this definition is not based on rigorous studies of the effect of different cut-offs for protection from damage.

For further work, it might be of interest to look at the predictive effect of a range of durations of remission on outcome. It stands to reason that remission maintained for a longer duration is 'better' than one maintained for only a short period, but it would be useful to know if achieving remission for shorter periods of time (1 or 2 years, or other arbitrary cut-offs) is also associated with significantly better outcomes. Use of shorter durations would facilitate implementing the study of remission in SLE in clinical research. Ultimately, the real question is how much additional information is imparted by adding the increasing time of observation; 
these kinds of questions could be addressed in the Padua cohort and in many other cohorts as well.

The work presented here also illustrates a sound approach to defining remission, namely, by testing the impact that various definitions may have on 'hard', long-term outcomes, such as damage which was used in this case. Other possibilities would be to study death or lupus flares. Whatever outcomes are chosen, a suitable study protocol for such analyses would have to be developed, with appropriately selected inclusion criteria, and well-defined frequency and quality of follow-up. Fortunately, there are several registries around the world-such as the one in Padua-that could be used for such analyses, as well as rich datasets from clinical trials with long-term extension protocols.

The finding by Zen et al that patients in clinical remission with and without serological activity behaved similarly could be explained by the fact that only anti-DNA antibodies were considered. It may be of importance to consider the entire antinuclear antibody (ANA) profile, since other antibodies such as anti-RNA binding protein antibodies can contribute to lupus pathogenesis, possibly via effects on interferon production, and could be included in future analyses. Likewise, biomarkers such as the interferon signature might be of interest in this regard.

In summary, the existence of many definitions of remission in SLE over the years underscores the need for unification of the research field in this regard. While an international initiative is currently underway to make this possible, data such as presented by Zen et al will be of great importance to further these efforts, enabling better studies and, as a result, better understanding, treatments and outcomes for this elusive disease.

Contributors RFvV wrote the first draft and all authors reviewed and revised it, and all authors have agreed on the final version.

Competing interests None declared.

Provenance and peer review Commissioned; externally peer reviewed.

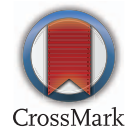

To cite van Vollenhoven RF, Voskuyl A, Morand E, et al. Ann Rheum Dis 2015;74:2103-2106.
Received 21 August 2015

Revised 27 September 2015

Accepted 29 September 2015

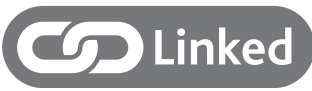

http://dx.doi.org/10.1136/annrheumdis-2015207347

Ann Rheum Dis 2015:74:2103-2106.

doi:10.1136/annrheumdis-2015-208231

\section{REFERENCES}

1 Pinals RS, Masi AT, Larsen RA. Preliminary criteria for clinical remission in rheumatoid arthritis. Arthritis Rheum 1981;24:1308-15.

2 Felson DT, Smolen JS, Wells G, et al. American College of Rheumatology/European League against Rheumatism provisional definition of remission in rheumatoid arthritis for clinical trials. Ann Rheum Dis 2011;70:404-13

3 Best WR, Becktel JM, Singleton JW, et al. Development of a Crohn's disease activity index. National Cooperative Crohn's Disease Study. Gastroenterology 1976;70:439-44.

4 Saag KG, Teng GG, Patkar NM, et al. American College of Rheumatology 2008 recommendations for the use of nonbiologic and biologic disease-modifying antirheumatic drugs in rheumatoid arthritis. Arthritis Rheum 2008;59:762-84.

5 Smolen JS, Landewé R, Breedveld FC, et al. EULAR recommendations for the management of rheumatoid arthritis with synthetic and biological disease-modifying antirheumatic drugs: 2013 update. Ann Rheum Dis 2014;73:492-509.

6 Smolen JS, Breedveld FC, Burmester GR, et al. Treating rheumatoid arthritis to target: 2014 update of the recommendations of an international task force. Ann Rheum Dis 2015. Published Online First 12 May 2015. doi:10.1136/annrheumdis-2015-207524

7 Lightfoot RW Jr, Hughes GR. Significance of persisting serologic abnormalities in SLE. Arthritis Rheum 1976;19:837-43.

8 Steiman AJ, Urowitz MB, Ibañez D, et al. Prolonged clinical remission in patients with systemic lupus erythematosus. J Rheumato/ 2014;41:1808-16.

9 Dubois EL. Systemic lupus erythematosus: recent advances in its diagnosis and treatment. Ann Intern Med 1956:45:163-84.

10 Dubois EL, Tuffanelli DL. Clinical manifestations of systemic lupus erythematosus. Computer analysis of 520 cases. JAMA 1964;190:104-11.

11 Gladman DD, Urowitz MB, Keystone EC. Serologically active clinically quiescent systemic lupus erythematosus: a discordance between clinical and serologic features. Am J Med 1979:66:210-15.

12 Tozman EC, Urowitz MB, Gladman DD. Prolonged complete remission in previously severe SLE. Ann Rheum Dis 1982;41:39-40.

13 Heller CA, Schur PH. Serological and clinical remission in systemic lupus erythematosus. J Rheumatol 1985:12:916-18.

14 LeBlanc BA, Urowitz MB, Gladman OD. Serologically active, clinically quiescent systemic lupus erythematosus-long-term follow-up. J Rheumatol 1994;21:174-5.
15 Drenkard C, Villa AR, Garcia-Padilla C, et al. Remission of systematic lupus erythematosus. Medicine (Baltimore) 1996;75:88-98.

16 Barr SG, Zonana-Nacach A, Magder LS, et al. Patterns of disease activity in systemic lupus erythematosus. Arthritis Rheum 1999:42:2682-8.

17 Formiga F, Moga I, Pac M, et al. High disease activity at baseline does not prevent a remission in patients with systemic lupus erythematosus. Rheumatology (Oxford) 1999;38:724-7.

18 Swaak AJ, van den Brink HG, Smeenk RJ, et al. Systemic lupus erythematosus: clinical features in patients with a disease duration of over 10 years, first evaluation. Rheumatology (Oxford) 1999:38:953-8.

19 Urowitz MB, Feletar M, Bruce IN, et al. Prolonged remission in systemic lupus erythematosus. J Rheumatol 2005;32:1467-72.

20 Nossent J, Kiss E, Rozman B, et al. Disease activity and damage accrual during the early disease course in a multinational inception cohort of patients with systemic lupus erythematosus. Lupus 2010;19:949-56.

21 Steiman AJ, Gladman DD, Ibañez D, et al. Prolonged serologically active clinically quiescent systemic lupus erythematosus: frequency and outcome. J Rheumato 2010;37:1822-7.

22 Conti F, Ceccarelli F, Perricone C, et al. Flare, persistently active disease, and serologically active clinically quiescent disease in systemic lupus erythematosus: a 2-year follow-up study. PLoS One 2012:7:e45934.

23 Franklyn K, Hoi A, Nikpour M, et al. The need to define treatment goals for systemic lupus erythematosus. Nat Rev Rheumatol 2014;10:567-71.

24 van Vollenhoven RF, Mosca M, Bertsias G, et al. Treat-to-target in systemic lupus erythematosus: recommendations from an international task force. Ann Rheum Dis 2014;73:958-67.

25 van Vollenhoven RF, Aranow C, Bertsias G, et al. Remission in SLE: consensus findings from a large international panel on definitions of remission in SLE (DORIS). Ann Rheum Dis 2015;73:671.

26 Morand E, Franklyn K, Lau CS, et al. Consensus definition and preliminary validation of a low disease activity state in systemic lupus erythematosus. Poster, ACR Annual Congress, 2013.

27 Mina R, Klein-Gitelman MS, Ravelli $A$, et al. Inactive disease and remission in childhood-onset systemic lupus erythematosus. Arthritis Care Res (Hoboken) 2012;64:683-93.

28 Steiman AJ, Gladman DD, Ibañez D, et al. Outcomes in patients with systemic lupus erythematosus with and without a prolonged serologically active clinically quiescent period. Arthritis Care Res (Hoboken) 2012;64:511-8

29 Györi N, Chatzidionysiou A, Magder L, et al. Disease activity patterns over time in patients with SLE-a retrospective descriptive analysis of the Hopkins lupus cohort. Ann Rheum Dis 2015;74:798.

30 Zen $\mathrm{M}$, laccarino L, Gatto $\mathrm{M}$, et al. Prolonged remission in Caucasian patients with SLE: prevalence and outcomes. Ann Rheum Dis 2015;74:2117-22.

31 Gladman D, Ginzler E, Goldsmith C, et al. The development and initial validation of the Systemic Lupus International Collaborating Clinics/ American College of Rheumatology damage index for systemic lupus erythematosus. Arthritis Rheum 1996:39:363-9. 